\title{
Nominata dos Avaliadores ad hoc 2018
}

Nominata dos Avaliadores que participaram da avaliação e revisão por pares feitas para o processo seletivo de artigos submetidos à revista Atualidade Teológica em 2018 foram:

Alessandro Rodrigues Rocha

Alex Villas Boas Oliveira Mariano

Alfonso Garcia Rubio

André Luiz Rodrigues da Silva

Antônio Geraldo Cantarela

Antônio José de Almeida

Antônio Luiz Catelan

Arthur Francisco Juliatti dos Santos

Ceci Maria C. Baptista Mariani

Cesar Augusto Kuzma

Claiton André Kunz

Cláudio de Oliveira Ribeiro
PUC-Rio

PUC-PR

PUC-Rio

PUC-Rio

PUC-MG

PUC-PR

PUC-Rio

IFTAV

PUC-Campinas

PUC-Rio

FABAPAR

PUC-Campinas 
Claudio Vianney Malzoni

Clélia Peretti

David Mesquiati de Oliveira

Dorival Souza Barreto Júnior

Douglas Rodrigues da Conceição

Drance Elias da Silva

Eduardo Gross

Elildes Junio Macharete Fonseca

Élio Estanislau Gasda

Érico João Hammes

Francilaide de Queiroz Ronsi

Francisco das C. de Albuquerque

Francisco de Assis Costa Taborda

Geraldo De Mori

Geraldo Luiz Borges Hackmann

Gilcemar Hohemberger

Gustavo Correa Cola

Isidoro Mazzarolo

Jaldemir Vitório

Jesus Hortal Sànchez

João Décio Passos

Johan Konings

Josias da Costa Júnior

Júlio Cesar Bendinelli

Júlio Cezar Adam

Kenner Roger Cazzoto Terra

Leomar Antônio Brustolin

Lúcia Pedrosa de Pádua

Luís Henrique Eloy e Silva

Luiz Carlos Sureki

Luiz Carlos Susin

María Del Pilar Silveira
UNICAP

PUC-PR

Faculdade Unida de Vitória

UNIMONTES

UEPA

UNICAP

UFJF

STMBL

FAJE

PUC-RS

Faculdade São Bento-RJ

FAJE

FAJE

FAJE

PUC-RS

Faculdade São Bento-RJ

PUC-RS

FAJE

PUC-Rio

PUC-SP

FAJE

UEPA

IFTAV

EST

Faculdade Unida de Vitória

PUC-RS

PUC-Rio

FAJE

FAJE

PUC-RS

UPS 
Maria Teresa de Freitas Cardoso

PUC-Rio

Mario Antonio Sanches

PUC-PR

Mario de França Miranda

PUC-Rio

Matthias Grenzer

PUC-SP

Paulo César Barros

FAJE

Pedro Kuniharu Iwashita

PUC-SP

Roberlei Panasiewicz

PUC-MG

Salma Ferraz

UFSC

Silvana de Gaspari

UFSC

Teresa Messias

UCP

Vitor Galdino Feller

FACASC

Waldecir Gonzaga

PUC-Rio

704 ATeo, Rio de Janeiro, v. 22, n. 60, p. 702-704, set./dez.2018 